\section{Associação entre excesso de peso e hábito de fumar, Santarém, PA, 2007}

\section{Association between overweight and smoking habits, Santarém, PA, 2007}

\section{Naíza Nayla Bandeira de Sá'}

Erly Catarina Moura"l,III

' Faculdade de Nutrição, Instituto de Ciências da Saúde, Universidade Federal do Pará, Belém, PA.

"Núcleo de Pesquisas Epidemiológicas em Nutrição e Saúde da Universidade de São Paulo, São Paulo, SP, Brasil.

"'Secretaria de Vigilância em Saúde, Ministério da Saúde. Brasília, DF.

\section{Resumo}

Objetivo: Estudar associação entre excesso de peso e hábito de fumar. Metodologia: Estudo transversal desenvolvido por telefone numa amostra probabilística de adultos (Santarém /PA), em 2007. Variável desfecho foi excesso de peso, explanatória hábito de fumar e de confusão idade, escolaridade, união conjugal, estado nutricional prévio, abuso de bebidas alcoólicas, atividade física no lazer e padrão alimentar. Associação entre excesso de peso e demais variáveis foi investigada pelo teste do qui-quadrado e regressão de Poisson para o cálculo das razões de prevalência de excesso de peso conforme hábito de fumar, considerando-se três níveis de hierarquia: características sociodemográficas, estado nutricional prévio e padrão comportamental. Resultados: Verificou-se $40,6 \%$ de excesso de peso e $16,4 \%$ de fumantes. As variáveis associadas ao excesso de peso foram: maior idade, menor escolaridade, união conjugal estável, excesso de peso aos 20 anos e hábito de não consumo de refrigerante para ambos os sexos; não ativo no lazer para homens e hábito de fumar (atual e passado) para mulheres. As razões de prevalência de excesso de peso não apresentaram associação com hábito de fumar para homens, porém para mulheres houve tendência de maior prevalência de excesso de peso para fumantes atuais, chegando a 2,56 vezes mais do que para nunca fumantes e ex-fumantes. Conclusão: Este estudo constatou que a prevalência de excesso de peso foi maior para mulheres fumantes, comparativamente às ex-fumantes e nunca fumantes. Para os homens não se observou nenhuma associação entre excesso de peso e tabagismo.

Palavras-chave: Excesso de peso. Tabagismo. Adultos. Entrevista telefônica. Santarém. 


\section{Abstract}

Objective: To study the relationships between overweight and smoking habit. Methods: A cross-sectional study was developed in a random sample $(n=944)$ of adults $(\geq 18$ years of age) from Santarem (state of Pará, northern Brazil) in 2007, through telephone interviews. The outcome variable was overweight and the explanatory variable, smoking habit. Confounding variables were: age, race, schooling, marital status, smoking habit, nutritional status at 20 years of age, alcohol abuse, leisure physical activity, and diet. The association between overweight and other variables was investigated by the chi-square test and Poisson regression in order to calculate crude and adjusted prevalence ratios, for overweight according to smoking habit. Three levels of hierarchy were considered: socio-demographic characteristics, nutritional status, and behavior. Results: Data show $40.6 \%$ of overweight and $16.4 \%$ of smokers. Variables associated with overweight were: older age, low level of schooling, stable marital relationship, overweight at 20 years of age, and nonconsumption of soft drinks for both sexes; no physical activity in leisure time for men and smoking habit (present and past) for women. Prevalence ratios of overweight were not associated with smoking habits for men, but for women there was a trend toward a higher prevalence of overweight for current smokers, reaching 2.56 times more than among never smokers and former smokers. Conclusion: This study showed a higher risk of overweight among women smokers, comparatively to former and never smokers. For men there was no association between overweight and smoking habit.

Keywords: Overweight. Smoking. Adults. Telephone interview. Santarém.

\section{Introdução}

As doenças crônicas não transmissíveis (DCNT), principais causas de morbidade e mortalidade atual, têm como principais fatores de risco comportamentais o hábito de fumar e a obesidade. $\mathrm{O}$ excesso de peso tem aumentado drasticamente, chegando a alcançar dois bilhões de indivíduos com 15 anos ou mais de idade ${ }^{1}$.

Dados de 2007 mostraram que no Brasil a obesidade alcançou $43 \%$ da população adulta e o hábito de fumar atingiu aproximadamente 16,4\% desta. Em Belém, capital do Estado do Pará, o hábito de fumar atingiu $14,3 \%$ da população adulta, enquanto o excesso de peso alcançou $41,8 \%^{2}$.

Vários fatores se associam diretamente com o excesso de peso, entre eles a idade, o estado conjugal, a escolaridade e o sedentarismo. Alguns destes fatores também têm sido associados ao hábito de fumar e, conseqüentemente entre si, isto é entre hábito de fumar e excesso de peso ${ }^{3}$. Um dos grandes desestímulos para abandonar o hábito de fumar é o medo do ganho de peso $^{4}$, ainda que os benefícios para a saúde sejam muitos. Todavia, diversos estudos apontam associação entre IMC e hábito de fumar, embora os resultados ainda sejam controversos. Akbartabartoori et al. $(2005)^{5}$ encontraram menor IMC em fumantes atuais e ex-fumantes quando comparados aos não-fumantes, John et al. (2005) ${ }^{6}$ apontaram que os homens que fumavam 30 cigarros por dia tiveram 5 vezes mais chances de serem obesos quando comparados com os homens que nunca fumaram. Kalter-Leibovici et al. (2007) ${ }^{7}$ encontraram que a obesidade esteve associada significativamente com o hábito de fumar, enquanto o estudo de Peixoto et al. $(2007)^{8}$ demonstrou associação positiva entre IMC e não fumante, para as mulheres. Este estudo tem como objetivo estudar a associação entre excesso de peso e hábito de fumar, considerando fatores sociodemográficos e comportamentais em homens e mulheres em Santarém (PA). 


\section{Metodologia}

O estudo realizado foi do tipo transversal, a partir de dados obtidos no ano de 2007, por meio de inquérito telefônico, para o monitoramento de fatores de risco para doenças crônicas não transmissíveis. Trata-se de estudo de base populacional, realizado junto a 1.066 indivíduos com $18 \mathrm{e}$ mais anos de idade residentes em Santarém (PA). A amostra para o estudo foi obtida em duas etapas: sorteio sistemático de cinco mil linhas telefônicas fixas residenciais, que foram re-sorteadas e agrupadas em 25 réplicas de 200 números cada e, para cada residência que concordou em participar, sorteio de um morador adulto entre todos os moradores adultos da residência. Calculouse um mínimo de mil entrevistas para poder estimar com intervalo de confiança de $95 \%$ e erro máximo de cerca de cinco pontos percentuais na freqüência de qualquer fator na população estudada.

$\mathrm{O}$ inquérito telefônico constou de entrevista utilizando questionário eletrônico desenvolvido por Monteiro et al. (2005) ${ }^{9}$ a partir de modelos de outros sistemas ${ }^{10,11}$; contendo questões sobre: características demográficas e socioeconômicas, características do padrão de alimentação e de atividade física, peso e altura recordados, frequência do consumo de cigarros e de bebidas alcoólicas, auto-avaliação do estado de saúde do entrevistado e referência a diagnóstico médico anterior de hipertensão arterial, diabetes e colesterol elevado. Para este estudo foram utilizados dados de sexo, idade, cor, escolaridade, união conjugal, hábito de fumar, peso e altura recordados (aos 20 anos de idade e atual), consumo abusivo de bebidas alcoólicas, prática de atividade física suficiente no lazer e padrão alimentar. Foram excluídos: homens e mulheres que não informaram peso atual e/ou altura e mulheres grávidas, restando 944 sujeitos.

Considerou-se o excesso de peso como variável desfecho (dependente). Excesso de peso foi considerado quando IMC - (peso em quilos dividido pelo quadrado da altura em metros) fosse maior ou igual a $25 \mathrm{~kg} / \mathrm{m}^{2}$
(WHO, 1995) ${ }^{12}$, sendo classificado em sim ou não. A variável explanatória foi o hábito de fumar, categorizado em: nunca fumante, ex-fumante ou fumante atual. As possíveis variáveis de confusão investigadas foram: idade, cor, escolaridade, união conjugal, estado nutricional aos 20 anos, consumo abusivo de bebidas alcoólicas, atividade física suficiente no lazer e padrão alimentar. A idade foi classificada em seis faixas etárias: 18-24, 25-34, 35-44, 45-54, ou $\geq 55$ anos de idade; cor em duas categorias: branca/ amarela ou negra/parda; a escolaridade em três faixas: 0-4, 5-8 ou $\geq 9$ anos de estudo; a união conjugal em duas categorias: estável ou não estável; o estado nutricional prévio em duas categorias: excesso de peso ou não excesso de peso, sendo o peso auto-referido aos 20 anos de idade. O consumo abusivo de bebidas alcoólicas foi classificado em sim ou não quando havia ou não consumo de mais de cinco doses de bebida alcoólica para o homem e mais de quatro para a mulher numa mesma ocasião, pelo menos uma vez nos últimos trinta dias. A atividade física suficiente no lazer foi categorizada em sim ou não, considerando-se ativo o indivíduo que relatou realizar $\geq 30$ minutos $\mathrm{em} \geq 5$ dias na semana de atividade leve ou moderada ou $\geq 20$ minutos em $\geq 3$ dias na semana de atividade vigorosa; o padrão alimentar foi avaliado conforme baixo consumo de feijão, baixo consumo de frutas, legumes e verduras (ambos menor que cinco dias na semana), hábito de consumo de carne ou frango com gordura visível, hábito de consumo de leite com teor integral de gordura e alto consumo de refrigerantes ( $\geq 3$ dias na semana), todos categorizados em sim ou não.

Para obter estimativas gerais das variáveis estudadas para a população adulta do município foram utilizados três fatores de ponderação:

- número de adultos em cada residência;

- inverso do número de linhas telefônicas fixas em cada residência; $\mathrm{e}$

- razão entre a freqüência relativa de indivíduos da amostra estudada e da amostra do censo de 2000 em categorias sociodemográficas determinadas. 
O detalhamento da ponderação da amostra pode ser encontrado em outras publicações ${ }^{9,13}$.

Inicialmente, calculou-se a distribuição da população segundo as variáveis estudadas (total e por sexo), considerando-se intervalo de confiança de $95 \%$. A prevalência de excesso de peso foi calculada para todas as variáveis, separadamente por sexo, utilizando-se nível de significância de 5\% ( $p$ $<0,05$ ), conforme teste do qui-quadrado. As variáveis $\operatorname{com} p<0,20$ foram selecionadas para ajuste em análise multivariada por regressão de Poisson. Calcularam-se as razões de prevalência de excesso de peso conforme o hábito de fumar, brutas e ajustadas, considerando-se três níveis de hierarquia: características sociodemográficas, estado nutricional prévio e padrão comportamental. O modelo de análise hierárquica, metodologia aplicada em análise multivariada para avaliar o efeito de fatores de risco sobre o desfecho avaliado, busca otimizar a explicação de possíveis associações em estudos do tipo transversal. Foi utilizado nível de significância de 5\%. Para a análise dos dados foi utilizado o aplicativo STATA versão 9.2, utilizando-se comandos que levam em contar o caráter de amostra complexa.

Por se tratar de entrevista por telefone, o consentimento livre e esclarecido foi substituído pelo consentimento verbal, obtido por ocasião dos contatos telefônicos com os entrevistados. Este estudo foi aprovado pelo Comitê de Ética em Pesquisa para seres humanos do Instituto de Ciências da Saúde da Universidade Federal do Pará.

\section{Resultados}

A Tabela 1 mostra as características da população estudada conforme o sexo. A maior parte da população está compreendida na faixa etária de 18 a 34 anos, é de cor negra/parda, estudou de 5 a 8 anos, referiu união conjugal estável, nunca fumou, não apresentou excesso de peso aos 20 anos de idade, não consome bebida alcoólica de forma abusiva, não é suficientemente ativa no lazer, apresenta baixo consumo de feijão, baixo consumo de frutas, legumes e verduras, não tem hábito de consumir carne ou frango com gordura, usa leite com teor de gordura integral e não consome refrigerante regularmente. Quanto ao estado nutricional atual, verifica-se excesso de peso em $47,6 \%$ de homens e $32,8 \%$ das mulheres.

A Tabela 2 mostra a prevalência do excesso de peso na população estudada, segundo características sociodemográficas e fatores de risco para doenças crônicas não transmissíveis, por sexo. A prevalência de excesso de peso na população masculina aumenta com a idade, chegando a atingir $73,7 \%$ da população com idade $\geq 55$ anos; é maior entre os homens com escolaridade intermediária (entre 5 a 8 anos de estudo), naqueles com excesso de peso aos 20 anos de idade, nos não suficientemente ativos no lazer, naqueles com não hábito de consumo regular de refrigerante. Para o sexo feminino, o excesso de peso também aumenta com a idade, chegando a atingir $58,3 \%$ da população com idade $\geq 55$ anos; é maior entre as mulheres de menor escolaridade (entre 0 a 4 anos de estudo), naquelas que têm união conjugal estável, que já foram ou são tabagistas, nas mulheres com excesso de peso aos 20 anos de idade, nas que não têm hábito de consumo de refrigerante.

A razão de prevalência de excesso de peso em homens (Tabela 3) é maior para os ex-fumantes (modelo 1), mas sem significância estatística. Após ajuste para idade, cor, escolaridade e união conjugal (modelo 2), há tendência de redução, mas também sem significância estatística. Esta situação permanece após ajuste para os modelos 3 (modelo $2+$ excesso de peso aos 20 anos de idade) e 4 (modelo 3 + variáveis comportamentais), mostrando que o excesso de peso em homens não se associa com hábito de fumar. Para as mulheres, a razão de prevalência de excesso de peso tende a ser maior para a ex-fumante e aumenta mais para a fumante atual (modelo 1), porém sem significância estatística, mesmo após a inclusão das variáveis sociodemográficas. Todavia, após ajuste para excesso de peso aos 20 anos de idade e demais variáveis comportamen- 
Tabela 1 - Distribuição' (\%) e IC 95\%² da população adulta ( $\geq 18$ anos de idade) segundo características sociodemográficas e fatores de risco para doenças crônicas não transmissíveis por sexo, Santarém, 2007.

Table 1 - Distribution (\%) and $95 \%$ Cl of the adult population ( $\geq 18$ years old) according to socio-demographic variables and prevalence of risk factors for chronic non communicable diseases by sex, Santarém, 2007.

\begin{tabular}{|c|c|c|c|c|c|c|}
\hline \multirow{2}{*}{$\begin{array}{l}\text { Características sócio-demográficas e } \\
\text { fatores de risco }\end{array}$} & \multicolumn{2}{|c|}{ Total $(n=944)$} & \multicolumn{2}{|c|}{ Masculino $(n=365)$} & \multicolumn{2}{|c|}{ Feminino $(n=579)$} \\
\hline & $\%$ & IC 95\% & $\%$ & IC 95\% & $\%$ & IC 95\% \\
\hline \multicolumn{7}{|l|}{ Idade (anos) } \\
\hline $18-24$ & 27.7 & $(20.6-34.8)$ & 27.9 & $(17.1-38.7)$ & 27.5 & $(18.6-36.5)$ \\
\hline $25-34$ & 26.1 & $(19.2-33.1)$ & 26.1 & $(14.7-37.5)$ & 26.1 & $(18.8-33.3)$ \\
\hline $35-44$ & 19.0 & $(14.8-23.2)$ & 17.8 & $(11.2-24.4)$ & 20.4 & $(15.3-25.4)$ \\
\hline $45-54$ & 13.1 & $(10.0-16.3)$ & 13.3 & $(8.5-18.1)$ & 12.9 & $(8.8-16.9)$ \\
\hline$\geq 55$ & 14.1 & $(10.8-17)$. & 14.9 & $(9.7-20.0)$ & 13.1 & $(9.4-16.9)$ \\
\hline \multicolumn{7}{|l|}{ Cor } \\
\hline Branca/Amarela & 25.6 & $(20.2-30.9)$ & 18.1 & $(11.7-24.5)$ & 33.9 & $(26.1-41.8)$ \\
\hline Negra/Parda & 74.4 & $(69.1-79.8)$ & 81.9 & $(75.5-88.3)$ & 66.1 & $(58.2-73.9)$ \\
\hline \multicolumn{7}{|l|}{ Escolaridade (anos) } \\
\hline $0-4$ & 19.7 & $(13.6-25.7)$ & 23.1 & $(12.9-33.3)$ & 15.8 & $(10.5-21.2)$ \\
\hline $5-8$ & 49.0 & $(42.1-56.0)$ & 51.0 & $(40.2-61.8)$ & 46.8 & $(38.4-55.2)$ \\
\hline$\geq 9$ & 31.3 & $(26.7-36.0)$ & 25.9 & $(19.7-32.1)$ & 37.4 & $(31.0-43.8)$ \\
\hline União Conjugal Estável & 56.6 & $(49.9-63.3)$ & 60.6 & $(50.4-70.7)$ & 52.1 & $(44.1-60.2)$ \\
\hline \multicolumn{7}{|l|}{ Hábito de fumar } \\
\hline Nunca Fumante & 61.6 & $(54.9-68.3)$ & 51.3 & $(40.4-62.1)$ & 73.2 & $(65.7-80.7)$ \\
\hline Ex - Fumante & 22.0 & $(17.1-27.0)$ & 25.0 & $(17.3-32.7)$ & 18.7 & $(12.4-25.0)$ \\
\hline Fumante & 16.4 & $(10.4-22.3)$ & 23.7 & $(13.8-33.6)$ & 8.1 & $(2.8-13.3)$ \\
\hline Excesso de Peso aos 20 anos de idade ${ }^{3}$ & 12.4 & $(6.9-18.0)$ & 16.1 & $(7.1-25.2)$ & 7.8 & $(2.3-13.3)$ \\
\hline Excesso de Peso Atual ${ }^{3}$ & 40.6 & $(34.2-47.1)$ & 47.6 & $(36.9-58.2)$ & 32.8 & $(25.7-40.0)$ \\
\hline Consumo Abusivo de Bebidas Alcoólicas ${ }^{4}$ & 19.1 & $(12.4-25.8)$ & 32.2 & $(21.1-43.3)$ & 4.4 & $(0.7-8.1)$ \\
\hline Não Ativo no Lazer ${ }^{5}$ & 85.9 & $(81.9-89.9)$ & 81.3 & $(74.3-88.2)$ & 91.1 & $(87.4-94.9)$ \\
\hline Baixo Consumo de feijão $^{6}$ & 67.9 & $(61.1-74.7)$ & 67.6 & $(56.9-78.2)$ & 68.2 & $(60.2-76.2)$ \\
\hline $\begin{array}{l}\text { Baixo Consumo de frutas, legumes e } \\
\text { verduras }{ }^{6}\end{array}$ & 91.1 & $(86.3-95.8)$ & 90.9 & $(83.4-98.3)$ & 91.2 & $(85.6-96.8)$ \\
\hline $\begin{array}{l}\text { Hábito de Consumo de carne/frango com } \\
\text { gordura visível }\end{array}$ & 33.9 & $(26.7-41.1)$ & 42.6 & $(31.5-53.6)$ & 24.2 & $(15.9-32.4)$ \\
\hline $\begin{array}{l}\text { Consumo de leite com teor integral de } \\
\text { gordura }\end{array}$ & 70.6 & $(63.6-77.6)$ & 70.9 & $(59.7-82.1)$ & 70.3 & $(62.4-78.3)$ \\
\hline Refrigerante ( $>3$ dias/semana) & 42.4 & $(35.2-49.5)$ & 51.6 & $(40.8-62.3)$ & 32.0 & $(24.3-39.7)$ \\
\hline \multicolumn{7}{|c|}{ 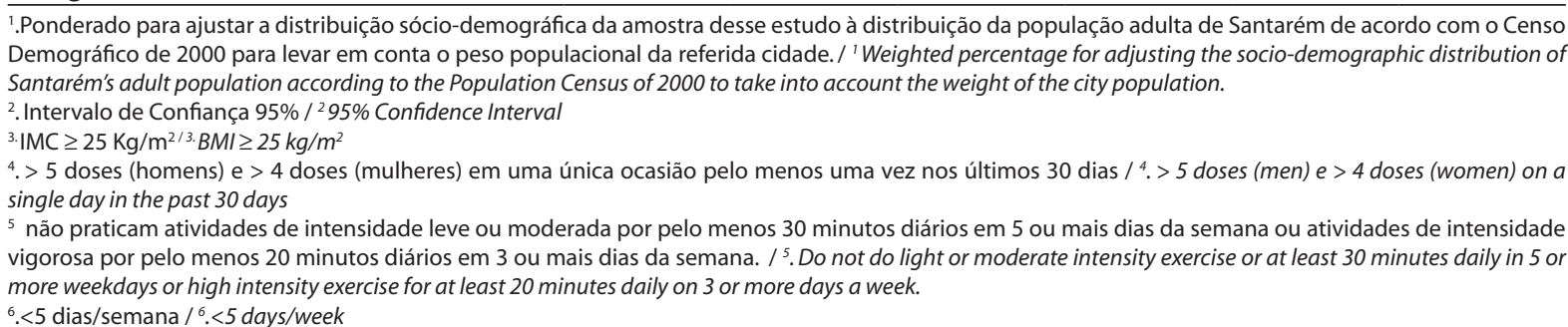 } \\
\hline
\end{tabular}

tais, a razão de prevalência de excesso de peso passa a ser significativamente maior para a fumante atual, isto é mulheres com hábito de fumar têm 2,56 vezes mais chance de apresentarem excesso de peso do que as nunca fumantes e ex-fumantes.

\section{Discussão}

O objetivo deste estudo foi verificar a associação entre excesso de peso e hábito de fumar na população adulta de Santarém, tendo sido observada associação entre o 
Tabela 2 - Prevalência' (\%) do excesso de peso da população adulta ( $\geq 18$ anos de idade) segundo características sociodemográficas e fatores de risco para doenças crônicas não transmissíveis, por sexo, Santarém, 2007.

Table 2 - Distribution (\%) of overweight in the adult population (> 18 years old) according to sociodemographic variables and prevalence of risk factors for chronic diseases by sex, Santarém, 2007.

\begin{tabular}{|c|c|c|c|c|}
\hline Características sócio-demográficas e fatores de & & lino & & ino \\
\hline risco & $\%$ & $p$ & $\%$ & $p$ \\
\hline Idade (anos) & & $<0.001$ & & $<0.001$ \\
\hline $18-24$ & 20.1 & & 19.6 & \\
\hline $25-34$ & 44.9 & & 19.4 & \\
\hline $35-44$ & 58.8 & & 46.6 & \\
\hline $45-54$ & 66.0 & & 40.8 & \\
\hline$\geq 55$ & 73.7 & & 58.3 & \\
\hline Cor & & 0.109 & & 0.523 \\
\hline Branca/Amarela & 59.9 & & 38.6 & \\
\hline Negra/Parda & 44.8 & & 29.9 & \\
\hline Escolaridade (anos) & & 0.001 & & $<0.001$ \\
\hline $0-4$ & 44.5 & & 45.2 & \\
\hline $5-8$ & 53.5 & & 36.7 & \\
\hline$\geq 9$ & 38.6 & & 22.7 & \\
\hline União Conjugal & & $<0.001$ & & $<0.001$ \\
\hline Não estável & 46.8 & & 20.9 & \\
\hline Estável & 48.1 & & 43.8 & \\
\hline Hábito de fumar & & 0.337 & & $<0.001$ \\
\hline Nunca Fumante & 45.7 & & 28.3 & \\
\hline Ex - Fumante & 53.1 & & 44.8 & \\
\hline Fumante & 45.7 & & 46.4 & \\
\hline Excesso de Peso aos 20 anos de idade ${ }^{2}$ & & $<0.001$ & & $<0.001$ \\
\hline Não & 50.9 & & 31.9 & \\
\hline Sim & 65.2 & & 78.3 & \\
\hline Consumo Abusivo de Bebidas Alcoólicas ${ }^{3}$ & & 0.438 & & 0.759 \\
\hline Não & 49.2 & & 33.4 & \\
\hline Sim & 44.1 & & 20.1 & \\
\hline Ativo no Lazer ${ }^{4}$ & & 0.004 & & 0.166 \\
\hline Não & 51.1 & & 30.7 & \\
\hline Sim & 32.3 & & 54.9 & \\
\hline Baixo Consumo de feijão ${ }^{4}$ & & 0.209 & & 0.734 \\
\hline Não & 47.7 & & 34.9 & \\
\hline Sim & 47.3 & & 28.4 & \\
\hline Baixo Consumo de frutas, legumes e verduras ${ }^{5}$ & & 0.704 & & 0.548 \\
\hline Não & 39.2 & & 28.1 & \\
\hline Sim & 48.4 & & 33.3 & \\
\hline $\begin{array}{l}\text { Hábito de Consumo de carne/frango com } \\
\text { gordura visível }\end{array}$ & & 0.064 & & 0.437 \\
\hline Não & 57.2 & & 33.0 & \\
\hline Sim & 34.6 & & 32.2 & \\
\hline Consumo de leite com teor integral de gordura & & 0.141 & & 0.156 \\
\hline Não & 50.6 & & 26.0 & \\
\hline Sim & 46.3 & & 35.7 & \\
\hline Refrigerante (>3 dias/semana) & & $<0.001$ & & 0.005 \\
\hline Não & 57.9 & & 36.0 & \\
\hline 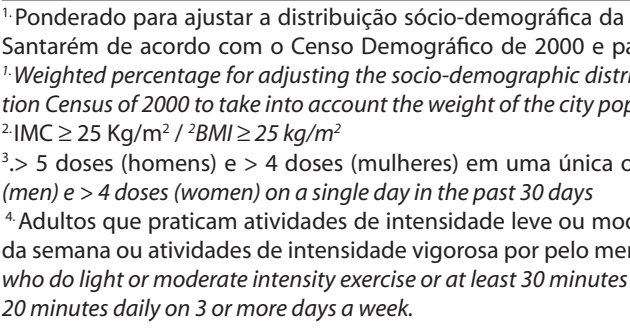 & $\begin{array}{l}\text { tra dess } \\
\text { var em } \\
n \text { of San } \\
\text { on. } \\
\text { o pelo r } \\
\text { a por pe } \\
\text { o minut }\end{array}$ & $\begin{array}{l}\text { udo à distri } \\
\text { o peso po } \\
\text { 's adult pop } \\
\text { s uma vez r } \\
\text { enos } 30 \mathrm{mir} \\
\text { rios em } 3 \text { o }\end{array}$ & $\begin{array}{l}\text { Iltimos } 3 \\
\text { s diários } \\
\text { is dias d }\end{array}$ & $\begin{array}{l}\text { cão adulta de } \\
\text { rida cidade. } \\
\text { to the Popula- } \\
\text { is } /{ }^{3}>5 \text { doses } \\
\text { ou mais dias } \\
\text { lana. } /{ }^{4} \text { Adults } \\
\text { ise for at least }\end{array}$ \\
\hline
\end{tabular}


Tabela 3 - Razão de prevalência (RP), bruta e ajustada, de excesso de peso segundo hábito de fumar por sexo, Santarém (PA), 2007.

Table 3- Crude and adjusted prevalence ratio (PR) of overweight according to smoking habits by sex, Santarém (PA), 2007

\begin{tabular}{|c|c|c|c|c|c|c|}
\hline \multirow[t]{2}{*}{ Hábito de fumar } & \multicolumn{3}{|c|}{ Masculino } & \multicolumn{3}{|c|}{ Feminino } \\
\hline & $\mathrm{RP}$ & IC 95\% & $p$ & $\mathrm{RP}$ & IC 95\% & $p$ \\
\hline Modelo 1 & & & 0.920 & & & 0.067 \\
\hline Nunca Fumante & 1 & & & 1 & & \\
\hline Ex-Fumante & 1.16 & $(0.74-1.83)$ & & 1.59 & $(0.96-2.60)$ & \\
\hline Fumante Atual & 1 & $(0.53-1.88)$ & & 1.64 & $(0.76-3.54)$ & \\
\hline Modelo 2 & & & 0.910 & & & 0.062 \\
\hline Nunca Fumante & 1 & & & 1 & & \\
\hline Ex - Fumante & 0.79 & $(0.54-1.14)$ & & 1.30 & $(0.72-2.34)$ & \\
\hline Fumante Atual & 1.05 & $(0.65-1.69)$ & & 1.78 & $(0.92-3.45)$ & \\
\hline \multicolumn{7}{|l|}{ Modelo 3} \\
\hline Nunca Fumante & 1 & & 0.576 & 1 & & 0.001 \\
\hline Ex - Fumante & 0.65 & $(0.40-1.05)$ & & 1.34 & $(0.89-2.02)$ & \\
\hline Fumante Atual & 0.95 & $(0.51-1.77)$ & & 2.43 & $(1.50-3.95)$ & \\
\hline Modelo 4 & & & 0.898 & & & 0.001 \\
\hline Nunca Fumante & 1 & & & 1 & & \\
\hline Ex-Fumante & 0.75 & $(0.50-1.13)$ & & 1.29 & (0.84-1.98) & \\
\hline Fumante Atual & 1.08 & $(0.68-1.72)$ & & 2.56 & $(1.58-4.14)$ & \\
\hline
\end{tabular}

Modelo 1: RP bruta / Model 1: crude PR

Modelo 2 para homens: RP ajustada para idade, cor, escolaridade e união conjugal / Model 2 for men: PR adjusted for age, race, schooling and marital relationship

Modelo 2 para mulheres: RP ajustada para idade, escolaridade e união conjugal / Model 2 for women: PR adjusted for age, schooling and marital relationship

Modelo 3: RP ajustada para variáveis do Modelo 2 + excesso de peso aos vinte anos de idade / Model 3: adjusted for variables in the PR model $2+$ overweight at twenty years of age

Modelo 4 para homens: RP ajustada para variáveis do Modelo 3 + ativo no lazer, consumo de carne ou frango com gordura visível, consumo de leite integral e consumo de refrigerante. / Model 4 for men: PR adjusted for variables of the Model 3 + active leisure, consumption of chicken or meat with visible fat, consumption of whole milk and consumption of soft drinks

Modelo 4 para mulheres: RP ajustada para variáveis do Modelo 3 + ativo no lazer, consumo de leite integral e consumo de refrigerante. / Model 4 for women: PR adjusted for variables of the Model 3 + active leisure, consumption of whole milk and of soft drinks.

desfecho estudado (excesso de peso) e o hábito de fumar para as mulheres, mas não para os homens. A razão de prevalência de excesso de peso, após ajuste para as variáveis sociodemográficas, excesso de peso aos 20 anos de idade e variáveis comportamentais, foi 2,56 vezes maior entre as mulheres fumantes do que entre as ex-fumantes e nunca fumantes.

Estudo semelhante realizado por Gigante et al. (1997) ${ }^{14}$, envolvendo 1.035 indivíduos de 20 a 69 anos de idade residentes em Pelotas (RS) e tendo a obesidade como desfecho e o hábito de fumar como um dos fatores de risco, apontou maior prevalência de obesidade entre mulheres ex-fumantes em relação às não fumantes e fumantes, não havendo diferença entre os homens. O estudo levou em consideração: variáveis demográficas, variáveis socioeconômicas, morbidades referidas, hábitos de alimentação e atividade física, a partir de modelo hierárquico. Das variáveis avaliadas neste estudo e no nosso, o estado nutricional prévio é a principal diferença, podendo explicar a diferença nos resultados, uma vez que, sem ajustar para as demais variáveis, nossos dados também apontam alta prevalência de excesso de peso em mulheres ex-fumantes, observando-se o mesmo para os homens.

Já estudo publicado de Lemos-Santos et al. ${ }^{15}$, avaliando o efeito do hábito de fumar no estado nutricional de 285 adultos entre 20 e 62 anos de idade, revelou que não houve 
diferença estatisticamente significante em relação ao índice de massa corporal dos ex-fumantes, fumantes e nunca fumantes em ambos sexos, porém a relação cinturaquadril foi maior para fumantes em relação aos não-fumantes, especialmente na faixa etária entre 20-39 anos.

Estudo transversal realizado com 7.124 adultos residentes na Alemanha, provenientes de uma amostra aleatória estratificada e traçada a partir das fichas de endereços do quais todos os residentes alemães entre 18 e 79 anos puderam ser incluídos, revelou que a prevalência de excesso de peso (préobesidade e obesidade) foi maior entre homens ex-fumantes e esteve diretamente associada ao número de cigarros fumados por dia, quando comparados com homens fumantes e não fumantes, mas não para as mulheres ${ }^{16}$. Os autores consideraram o estado nutricional como desfecho e o hábito de fumar como variável explanatória, ajustando para idade, escolaridade, atividade física e consumo de bebidas alcoólicas, mas não estado nutricional anterior.

Canoy e col. (2005) $)^{17}$ realizaram estudo do tipo multicêntrico de base populacional, com 21.828 indivíduos adultos com idade entre 45 e 79 anos, residentes em Norfolk, Reino Unido. Os autores obtiveram que a razão cintura-quadril, após ajuste para a idade, IMC, consumo de bebidas alcoólicas, ingestão calórica total, atividade física e escolaridade, foi maior entre os fumantes e menor entre os nunca fumantes, em relação aos ex-fumantes.

Akbartabartoori e col. (2005) $)^{5}$, em estudo do tipo transversal realizado com 9.047 adultos (16 a 74 anos de idade), apontaram menor IMC entre os fumantes e maior IMC entre os ex-fumantes, quando comparados aos não fumantes, o que se manteve após ajuste para idade, classe social, atividade física e consumo de bebidas alcoólicas. Quanto à faixa etária, a prevalência de tabagismo foi mais alta nos obesos e mais baixa nos indivíduos com IMC entre $25 \mathrm{e}$ $29,9 \mathrm{Kg} / \mathrm{m}^{2}$.

Estudo do tipo transversal, conduzido por Peixoto e col. $(2007)^{8}$ na cidade de
Goiânia, Estado de Goiás, em 2001, com 1.252 indivíduos com 20 a 64 anos de idade, encontrou IMC maior para as mulheres ex-fumantes quando comparadas às não fumantes após ajuste para idade, altura e estado conjugal, mas não para os homens, embora a associação fosse positiva para ambos os sexos na análise bivariada.

Estudo realizado por Yore e colaboradores (2007) ${ }^{18}$ com 8.467 adultos com 20 ou mais anos de idade, participantes da Pesquisa Nacional de Saúde e Nutrição nos Estados Unidos entre os anos de 1999 e 2002, apontou que não fumantes com hábito de assistir quatro ou mais horas de televisão por dia apresentaram maior prevalência de excesso de peso (pré-obesidade e obesidade) do que não fumantes com hábito de assistir menos de uma hora de televisão por dia, mas não houve diferença para os fumantes. Usando o hábito de fumar como ajuste, o hábito de assistir televisão mais do que duas horas por dia explicou o excesso de peso.

Como se pode ver, poucos são os estudos sobre estado nutricional e hábito de fumar, sendo que as variáveis levantadas e as técnicas de análise são distintas, o que dificulta a comparabilidade entre eles. De modo geral, os resultados são bastante heterogêneos. Todavia, nenhum dos estudos encontrados faz referência ao estado nutricional anterior, que é um dos determinantes do estado nutricional atual, isto é, jovens com excesso de peso têm maior probabilidade de se tornarem adultos com excesso de peso ${ }^{19}$. A não inclusão desta variável em estudos sobre estado nutricional atual e hábito de fumar pode levar a conclusões equivocadas, uma vez que o excesso de peso atinge cerca de $50 \%$ da população. Um dos limites desta análise é não ter avaliado a idade de início do tabagismo e o estado nutricional da época, o que não foi possível devido ao desenho transversal.

Outra limitação deste estudo foi a de que as variáveis utilizadas para a classificação de excesso de peso pelo IMC, isto é, peso e altura, foram auto-referidas, podendo ser super ou subestimadas pelos entrevistados. Peixoto et al. (2006) ${ }^{20}$ em estudo transversal 
de base populacional, realizado em Goiânia, capital do Estado de Goiás, no ano de 2001, junto a 1.023 indivíduos de 20 a 64 anos, constataram que os resultados encontrados para a prevalência de obesidade com peso e altura referidos foram semelhantes aos obtidos pelo peso e altura aferidos, o que torna confiável, também, os dados apresentados neste trabalho.

O instrumento de coleta de dados (inquérito telefônico) utilizado no presente levantamento difere dos estudos discutidos acima, o que evidencia outra limitação deste estudo, esta por excluir indivíduos que residem em domicílios sem telefonia fixa, porém, foram utilizados pesos para corrigir a super ou sub-representação da amostra, tendo como referência a composição sociodemográfica da população adulta da cidade de Santarém no Censo Demográfico mais recente. As freqüências obtidas neste estudo - excesso de peso ( $47,6 \%$ em homens e $32,8 \%$ em mulheres) e tabagismo $(23,7 \%$ em homens e $8,1 \%$ em mulheres) - são corroboradas por outros estudos de base populacional desenvolvidos no país ${ }^{2,21-23}$, bem como as associações identificas entre

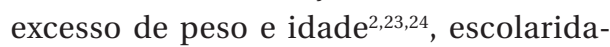
$\mathrm{de}^{9,25,26}$, tipo de união conjugal ${ }^{27,28}$, atividade física no lazer ${ }^{26} \mathrm{e}$, principalmente, excesso de peso aos 20 anos de idade ${ }^{29,30}$.

Utilizou-se estudo do tipo transversal, que tem como limitação o fato das medições serem feitas no mesmo momento, espaço e tempo, não podendo distinguir causa-efeito do problema. Ressalta-se, entretanto, que o objetivo deste trabalho foi verificar a associação entre excesso de peso e hábito de fumar, e que o uso de modelos hierárquicos é uma aproximação usada para estudos transversais no sentido de explicar possíveis associações.

Considerando que o ganho de peso após a cessação do tabagismo pode ser uma barreira na luta contra o fumo, que os estudos sobre este assunto são poucos e controversos, que este estudo aponta o estado nutricional prévio e a necessidade de analise de outras variáveis nesta relação, recomenda-se novos estudos mais detalhados sobre associação entre estado nutricional e hábito de fumar.

\section{Referências}

1. World Health Organization. Diet, Nutrition and the Prevention of Chronic Diseases. Report FAO/WHO Expert Consulation. WHO technical report series. Geneva: WHO; 2003.

2. Ministério da Saúde. VIGITEL 2007 - Vigilância de fatores de risco e proteção para Doenças Crônicas por Inquérito Telefônico. Brasília: MS; 2008.

3. Brown A, Siahpush M. Risk factors for overweight and obesity: results from the 2001 national health survey. Public Health 2007; 121(8): 603-13.

4. Escola Nacional de Saúde Pública Sérgio Arouca (on line), 2007. Abordagem do hábito de fumar. Disponível em www.ensp.fiocruz.br/eventos_novo/dados/arq 6485. ppt [Acessado em 10 de outubro de 2008]

5. Akbartabartoori M, Lean ME, Hankey CR. Relationships between cigarette smoking, body size and body shape. Int J Obes 2005; 29(2): 236-43.

6. Jonh U, Hanke M, Rumpf HJ, Thyrian JR. Smoking status, cigarettes per day, and their relationship to overweight and obesity among former and current smokers in a national adult general population sample. Int J Obes 2005; 29: 1289-94.
7. Kalter-Leibovic O, Atamna A, Lubin F, Alpert G, Keren MG, et al. Obesity among Arabs and Jews in Israel: A Population-Based Study. Isr Med Assoc J 2007; 9: 525-30.

8. Peixoto MRG, Benício MHD, Jardim PCBV. The relationship between body mass índex and lifestyle in a Brazilian adult population: a cross-sectional survey. Cad. Saúde Pública 2007; 23(11):2694-2704.

9. Monteiro CA, Moura EC, Jaime PC, Lucca P, Florindo AA, Figueiredo ICR et al. Monitoramento de fatores de risco para doenças crônicas por entrevistas telefônicas. Rev Saúde Pública 2005; 39(1): 47-57.

10. Remington PL, Smith MY, Williamson DF, Anda RF, Gentry EM, Hogelin GC. Design, characteristics, and usefulness of state-based behavioral risk factor surveillance. Public Health Rep 1988; 103(4): 366-75.

11. World Health Organization. Summary: surveillance of risk factors for non communicable diseases. The WHO STEP wise approach. Geneva: WHO; 2001.

12. World Health Organization. Physical Status: the use and interpretation of anthropometry. Report of WHO expert committee. Geneva:WHO; 1995. 
13. Moura EC, Neto OLM, Malta DC, Moura L, Silva NN, Bernal R, et al. Vigilância de Fatores de Risco para Doenças Crônicas por Inquérito Telefônico nas capitais dos 26 estados brasileiros e no Distrito Federal (2006). Rev Bras Epidemiol 2008;11 (S1): 20-37.

14. Gigante PD, Barros FC, Post CLA, Olinto MTA. Prevalência de obesidade em adultos e seus fatores de risco. Rev Saúde Pública 1997; 31(3): 236-46.

15. Lemos-Santos, Ferreira MG, Gonçalves-Silva, Veras RM, Clóvis B. Hábito de fumar, composição corporal, distribuição da adiposidade e ingestão alimentar em fumantes, não-fumantes e ex-fumantes. Folha méd $2000 ; 119(3): 23-31$.

16. Jonh U, Hanke M, Rumpf HJ, Thyrian JR. Smoking status, cigarettes per day, and their relationship to overweight and obesity among former and current smokers in a national adult general population sample. Int J Obes 2005; 29: 1289-94.

17. Canoy D, Wareham N, Luben R, Welch A, Bingham S, Day N, et al. Cigarette Smoking and Fat Distribution in 21,828 British Men and Women: A Populationbased Study. Obes Res 2005; 13:1466-75.

18. Yore MM, Fulton JE, Nelson DE, Kohl HW. Cigarette smoking status and the association between media use and overweight and obesity. Am J Epidemiol 2007; 166(7): 795-802.

19. World Health Organization. The World Health Report Reducing Risks, Promoting Healthy Life. Geneva; 2002.

20. Peixoto MRG, Benício MHDA, Jardim PCBV. Validade do peso e altura referidos: o estudo de Goiânia. Rev Saúde Pública 2006; 40(6): 1065-72.

21. Ministério da Saúde. Secretaria de Vigilância em Saúde. Secretária de Atenção à Saúde. Instituto Nacional de Câncer. Inquérito Domiciliar sobre comportamentos de risco e morbidade referida de doenças e agravos não transmissíveis. Brasília: MS; 2003.

22. Ministério da Saúde e Instituto Nacional de Câncer. Inquérito Domiciliar sobre comportamentos de risco e morbidade referida de doenças e agravos não transmissíveis: Brasil, 15 capitais e Distrito Federal 2002-
2003. Rio de Janeiro: INCA; 2004.

23. Monteiro CA, Cavalcante TM, Moura EC, Claro RM, Szwarcwald CL. Population-based evidence of a strong decline in the prevalence of smokers in Brazil (19892003). Bull World Health Organ 2007; 85(7): 527-34.

24. Instituto Brasileiro de Geografia e Estatística. Pesquisa de orçamentos familiares 2002 - 2003. Análise da disponibilidade domiciliar de alimentos e do estado nutricional no Brasil. IBGE. Rio de Janeiro; 2004.

25. Carvalhaes MABL, Moura EC, Monteiro CA. Prevalência de fatores de risco para doenças crônicas: inquérito populacional mediante entrevistas telefônicas em Botucatu, São Paulo, 2004. Rev Bras Epidemiol 2008; 11(1): 14-16.

26. Peixoto MRG, Monego ET, Alexandre VP, Souza RGM, Moura EC. Monitoramento por entrevistas telefônicas de fatores de risco para doenças crônicas: experiência de Goiânia, Goiás, Brasil. Cad Saúde Pública 2008; 24(6): $1323-33$.

27. Brilmann M, Oliveira MS, Thiers VO. Avaliação da qualidade de vida relacionada à saúde na obesidade. Cad Saúde Col 2007; 15(1): 39-54.

28. Teichmann L, Olinto MTA, Costa JSD, Ziegler D. Fatores de risco associados ao sobrepeso e a obesidade em mulheres de São Leopoldo, RS. Rev Bras Epidemiol 2006; 9 (3): 360-73.

29. Gordon-Larsen P, Adair LS, Nelson MC, et al. Five-year obesity incidence in the transition period between adolescence and adulthood: the National Longitudinal Study of Adolescent Health1-3. Am J Clin Nutr 2004; 80: 569-75.

30. Wright CM, Parker L, Lamont D, Craft AW. Implications of childhood obesity for adult health: findings from thousand families cohort study. BMJ 2001; 323: 1280-4.

Recebido em: 22/04/09

Versão final reapresentada em: 19/08/09

Aprovado em: 28/08/09 